\title{
Molecular abundances in extragalactic sources
}

\author{
C. HENKEL and R. MAUERSBERGER \\ Max-Planck-Institut für Radioastronomie, Auf dem Hügel 69, D-5300 Bonn 1, \\ F.R. Germany
}

August 30, 1991

\begin{abstract}
Chemical properties of extragalactic molecular clouds are summarized. Column densities, relative abundances, and chemical implications are briefly discussed and references for more detailed studies are given. The need for model calculations is emphasized.
\end{abstract}

Key words: galaxies - molecules - abundances

\section{Introduction}

Until recently, molecular spectroscopy of extragalactic sources was confined to the observation of a few molecular species only. Since then, however, the situation has dramatically improved and 21 molecules, aside of $\mathrm{CO}$ and $\mathrm{H}_{2}$, have been detected to date. Previous articles summarizing these achievements are given by Henkel and Mauersberger (1991) and Henkel et al. (1991; see e.g. their Table 1). Here, we present a brief overview emphasizing measured column densities and abundances of the 21 species to provide an appropriate input for chemical models.

Most of the molecules are observed at mm-wavelengths and exhibit quasithermal emission. Their electric dipole moment is usually much larger than that of the commonly observed $\mathrm{CO}$ molecule, thus allowing the study of regions with typical densities $n\left(\mathrm{H}_{2}\right) \geq 10^{4} \mathrm{~cm}^{-3}$. One may distinguish four classes of sources which have been measured: (1) irregulars of the Local Group, (2) nearby spirals or interacting irregulars of nearby groups of galaxies, (3) a nearby elliptical, and (4) distant mergers at the upper end of the far infrared luminosity range of galaxies.

\section{Results and discussion}

\subsection{The LMC ANd SMC}

Johansson (1991) report the detection of $\mathrm{CN}, \mathrm{CS}, \mathrm{SO}, \mathrm{HCN}, \mathrm{HCO}^{+}, \mathrm{C}_{2} \mathrm{H}$, and $\mathrm{H}_{2} \mathrm{CO}$ toward the $\mathrm{HII}$ region $\mathrm{N} 159$ of the LMC. CS and $\mathrm{HCO}^{+}$are also observed in the SMC, toward N 19. A very preliminary analysis (assuming optically thin line emission, excitation temperatures between 5 and $50 \mathrm{~K}$, and a spatial extent of the emission relative to the $\mathrm{CO}$ cloud size similar to that in Galactic sources) suggests that abundances relative to $\mathrm{H}_{2}$ may be similar to those in 'local' molecular clouds. The exception is SO, which may have a very large relative abundance (X(SO) 3 $10^{-4} \ldots 10^{-2}$ ), but additional data are required to support the estimate. 


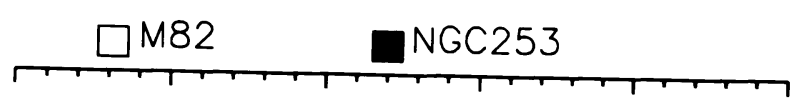

CN

CS

$\mathrm{SiO}$

HNCO

$\mathrm{CH}_{3} \mathrm{OH}$

$\mathrm{CH}_{3} \mathrm{CN}$

$\mathrm{CH}_{3} \mathrm{CCH}$

Fig. 1. Relative abundances for NGC 253 and $\mathrm{M} 82$. Adopted $\mathrm{H}_{2}$ column densities are $10^{23.2}$ and $10^{23.6} \mathrm{~cm}^{-2}$, respectively. For HNCO in the SW-hotspot of M 82 we assumed an excitation temperature of $10 \mathrm{~K}$, similar to that found in IC 342 and Maffei 2 (see Nguyen-Q-Rieu et al. 1991). For the other molecules, compare with Mauersberger and Henkel (1991; CN has been corrected).

\subsection{NGC 253 VERSUS M 82}

Among the nearby spirals and interacting irregulars the outstanding sources are NGC 253, Maffei 2, IC 342, M 82, and NGC 4945. The dense gas, emitting a large variety of molecular lines, is confined to the inner few $100 \mathrm{pc}$. NGC 253 and M 82 are the sources observed in greatest detail (e.g. Henkel and Mauersberger 1991; Henkel et al. 1991; Mauersberger and Henkel 1991; Mauersberger et al. 1991). Both have approximately the same distance. While NGC 253 contains a bar or ring (or both) with an angular distance between the two major hotspots of $9^{\prime \prime}(\sim 150 \mathrm{pc})$, the main lobes of the rotating ring in $\mathrm{M} 82$ are separated by $\sim 30^{\prime \prime}$. Because the source size of M 82 is larger than most telescope beams, Fig. 1 compares relative abundances between NGC 253 and the south-western lobe of M 82. Note that the $\mathrm{H}_{2}$ col- 


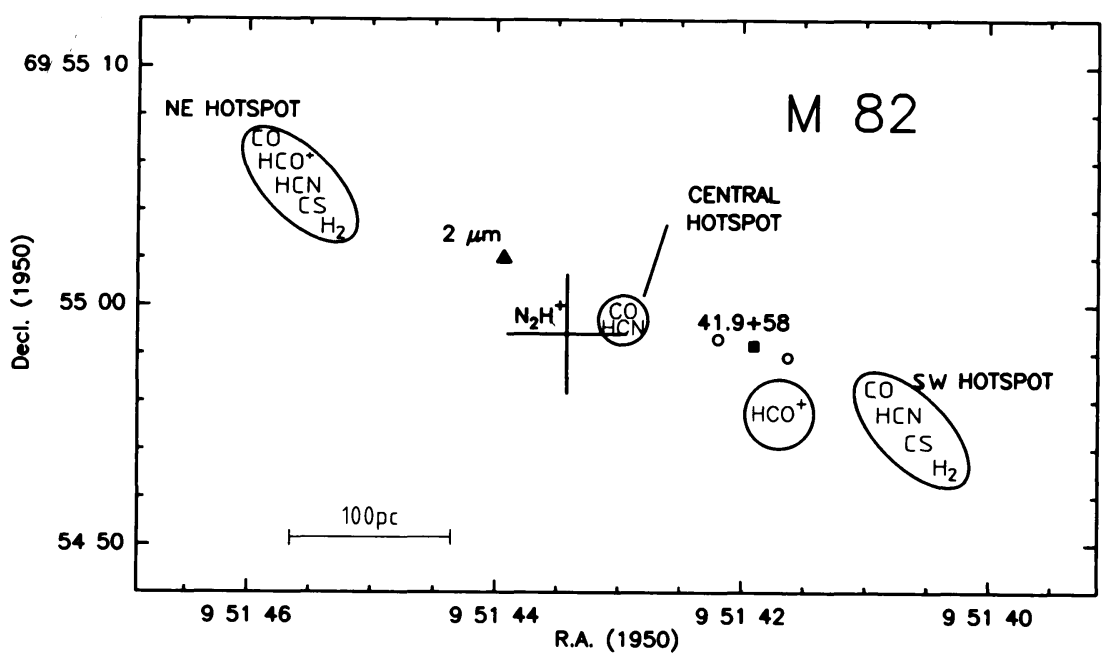

Fig. 2. A finding chart for molecular and continuum features in the nuclear region of M 82 (Mauersberger and Henkel 1991). Besides the molecular hotspots discussed in the text, the filled triangle marks the $2 \mu \mathrm{m}$ peak close to the kinematical center. The filled square marks the strongest radio continuum source, $41.9+58$, the open circles the location of the $\mathrm{OH}$ masers.

umn densities are poorly determined for such small regions and that relative abundances may be uncertain by at least an order of magnitude. Assuming, however, that the same $\mathrm{H}_{2}$ column density can be applied for all molecular species within a single source, a comparison of relative abundances can be made with high accuracy. There are at least two groups of molecules: $\mathrm{CN}$ and $\mathrm{CH}_{3} \mathrm{C}_{2} \mathrm{H}$ have similar relative abundances. $\mathrm{CS}$ is less abundant by an order of magnitude in M 82. The other molecules in M 82 may be even more 'underabundant' relative to NGC 253. All species have been observed in more than one transition so that non-LTE effects in a single transition are not responsible for the values given. In $\mathrm{M} 82$, the $\mathrm{CO} / \mathrm{HCN}$ line temperature ratio is extremely large (Nguyen-Q-Rieu et al. 1989), so that we have reason to suspect that $\mathrm{HCN}$ is another molecule which is 'underabundant' in M 82. Possible explanations in terms of different kinetic temperatures, $\mathrm{CH}_{3}^{+}$abundances, and non-steady state chemistry are outlined by Henkel et al. (1991) and Mauersberger et al. (1991). None of these interpretations is, however, satisfactory. Each of them only addresses a few of the observed species and the difference in the HNCO abundances (see Nguyen-Q-Rieu et al. 1991) remains entirely unexplained. 


\subsection{Spatial structure Within NuClear Regions}

Besides studying differences between various galaxies, there are a few sources where we can trace spatial abundance variations within a single nucleus. A possible example are the $\mathrm{HCN}$ and $\mathrm{HNC}$ profiles toward the central region of NGC 4945 (see Henkel et al. 1990) which show different lineshapes and may arise from different nuclear rings. This interpretation is, however, based on indirect arguments and is not yet supported by data which directly resolve the line emission.

A much more impressive case is hence the nuclear region of M 82 (see Fig. 2). We distinguish the main lobes (NE- and SW-hotspot), the central hotspot, and the $\mathrm{SW}-\mathrm{HCO}^{+}$-hotspot which is located closer to the nucleus than the 'associated' main lobe. Neutral molecules peak at the NE- and SWhotspots of the hypothesized rotating ring. Vibrationally excited $\mathrm{H}_{2}$ may arise from the inner molecular shell directly exposed to the active nuclear region (Lester et al. 1990). The density of the NE-hotspot, $n\left(\mathrm{H}_{2}\right) \sim 10^{5} \mathrm{~cm}^{-3}$ (Mauersberger and Henkel 1989), is large enough to cause a low $\mathrm{N}_{2} \mathrm{H}^{+}$abundance. In the SW-hotspot where densities are $>10^{5} \mathrm{~cm}^{-3}$ (Baan et al. 1990), both $\mathrm{N}_{2} \mathrm{H}^{+}$and $\mathrm{HCO}^{+}$do not exhibit noticeable peaks (Carlstrom 1988; Mauersberger and Henkel 1991). The central hotspot, however, exhibits most of the $\mathrm{N}_{2} \mathrm{H}^{+}$emission so that by analogy with Galactic clouds, the density should be at order $10^{4} \mathrm{~cm}^{-3}$ (Mauersberger and Henkel 1991). The $\mathrm{HCO}^{+}(1-0) / \mathrm{HCN}(1-0)$ or $\mathrm{HCO}^{+}(1-0) / \mathrm{CO}(1-0)$ intensity ratios drop considerably from the inner to the outer edges of the molecular lobes (Carlstrom 1988). One may speculate that this is due to shocks or a high cosmic ray flux at the inner edges of the molecular structure. The $\mathrm{HCO}^{+}$clump between the central and the SW-hotspot might represent dense molecular gas entrained by the the outflowing plasma from the nucleus.

\subsection{CEN A}

Toward this 'active' elliptical, $\mathrm{H}_{2}, \mathrm{CO}, \mathrm{OH}, \mathrm{HCN}, \mathrm{HNC}, \mathrm{HCO}^{+}, \mathrm{NH}_{3}, \mathrm{H}_{2} \mathrm{CO}$, and $\mathrm{C}_{3} \mathrm{H}_{2}$ have been detected (e.g. Bell and Seaquist 1988; Seaquist and Bell 1990; Eckart et al. 1990; Israel et al. 1990, 1991). There are at least four major components: (1) emission from the optically visible dust lane, only seen in $\mathrm{CO} ;(2)$ broad emission from the circumstellar gas observed in $\mathrm{CO}$ and $\mathrm{C}_{3} \mathrm{H}_{2} ;(3)$ narrow absorption lines at the systemic velocity, $\sim 550 \mathrm{~km} \mathrm{~s}^{-1}$, present in all detected molecular species except $\mathrm{H}_{2}$, and (4) narrow absorption lines at $\sim 600 \mathrm{~km} \mathrm{~s}^{-1}$ observed in $\mathrm{CO}, \mathrm{HCO}^{+}, \mathrm{OH}$, and perhaps $\mathrm{H}_{2} \mathrm{CO}$. The absorption lines sample gas along the line-of-sight toward the nuclear continuum source. The column densities are small (Seaquist and Bell 1990) so that either the clouds are small or the line-of-sight is intersecting the edges of the clouds only. A comparison of the column densities derived from 
the $\sim 550 \mathrm{~km} \mathrm{~s}^{-1}$ data (see Table 2 of Seaquist and Bell 1990) indicates 'normal' abundances when compared with Galactic dark cloud material. Note that the $\mathrm{NH}_{3}$ column density is as low as expected when only observing the edge of a clump. The absorption component at $\sim 606 \mathrm{~km} \mathrm{~s}^{-1}$ may represent gas falling toward the nucleus and arises from warm gas (Israel et al. 1991). Its $\mathrm{HCO}^{+} / \mathrm{CO}$ optical depth ratio appears to be large, indicating either enhanced cosmic ray ionization or shock induced processes. This component and the SW-HCO ${ }^{+}$-hotspot in $\mathrm{M} 82$ (Fig. 2) might be chemically related.

\subsection{Megamaser galaXies}

It is also possible to estimate $\mathrm{OH}$ column densities in megamaser galaxies, where the $18 \mathrm{~cm}$ main lines amplify the nuclear radio continuum. Assuming that the main lines have an optical depth ratio of 1.8:1 (this is the LTE intensity ratio in the optically thin case) and that excitation temperatures and continuum source covering factors are equal, the relative intensity of the 1665 and $1667 \mathrm{MHz}$ lines allows an estimate of the optical depth (Henkel and Wilson 1990; Henkel et al. 1991). In the likely case that the absolute values of of excitation temperatures are similar to the rotation temperature, $\sim 60 \mathrm{~K}$ (see Henkel et al. 1987), we obtain, irrespective of the detailed pumping mechanism, $\mathrm{OH}$ column densities of order $10^{18 \ldots 19} \mathrm{~cm}^{-2} ; \mathrm{X}(\mathrm{OH}) \sim 10^{-6}$. These large values might be caused by shocks, which should be common near the nuclei of merging systems, giving rise to megamaser emission.

The radiative $2 \mathrm{~cm}$ pumping mechanism suggested by Baan et al. (1986) to explain the $\mathrm{H}_{2} \mathrm{CO}$ maser in $\operatorname{Arp} 220$ requires $\mathrm{X}\left(\mathrm{H}_{2} \mathrm{CO}\right) \sim 10^{-8 \pm 1}$. This relative abundance is 'normal' by Galactic standards.

\section{Outlook}

We have shown that there exists already a large amount of data relevant for an analysis of chemical processes in extragalactic sources. While further drastic observational improvements are expected, it is remarkable that chemical models for the sources discussed above do not exist. It is yet too early for a thorough analysis of the Magellanic clouds. However, our knowledge of the nuclear regions of the other galaxies (see Sects. 2.2...2.5) is good enough to justify model calculations involving shock chemistry and/or a greatly enhanced flux of cosmic ray particles.

\section{References}

Baan. W.A.. Güsten, R., Haschick, A.D. 1986, Ap.J. 305, 830

Baan, W.A., Henkel, C., Schilke, P., Mauersberger, R., Güsten, R. 1990, Ap. J. 353, 132 Bell, M.B., Seaquist, E.R. 1988, Ap.J. 329, L17

Carlstrom, J.E. 1988, in 'Galactic and Extragalactic Star Formation', eds. R.E. Pudritz, M. Fich, Kluwer, p571 
Eckart, A., Cameron, H., Rothermel, H., Wild, W., Zinnecker, H., Rydbeck, G., Olberg, M., Wiklind, T. 1990, Ap.J. 363, 451

Henkel, C., Baan, W.A., Mauersberger, R. 1991, Astr. Ap. Rev. 3, 47

Henkel, C., Güsten, R., Baan, W.A. 1987, Astr. Ap. 185, 14

Henkel, C., Mauersberger, R. 1991, in 'Dynamics of Galaxies and Their Molecular Cloud Distributions', eds. F. Combes and F. Casoli, Kluwer, IA U Symp. 146, p195

Henkel, C., Whiteoak, J.B., L.-Å. Nyman, J. Harju 1990, Astr. Ap. 230, L5

Henkel, C., Wilson, T.L. 1990, Astr. Ap. 229, 431

Israel, F.P., van Dishoeck, E.F., Baas, F., Koorneef, J., Black, J.H., de Graauw, T. 1990, Astr. Ap. 227, 342

Israel, F.P., van Dishoeck, E.F., Baas, F., de Graauw, T., Phillips, T.G. 1991, Astr. Ap. 245, L13

Johansson, L.E.B. 1991, in 'Dynamics of Galaxies and Their Molecular Cloud Distributions', eds. F. Combes and F. Casoli, Kluwer, IAU Symp. 146, p1

Mauersberger, R., Henkel, C. 1989, Astr. Ap. 223, 79

Mauersberger, R., Henkel, C. 1991, Astr. Ap. 245, 457

Mauersberger, R., Henkel, C., Walmsley, C.M., Sage, L.J., Wiklind, T. 1991, Astr. Ap. 247, 307

Nguyen-Q-Rieu, Henkel, C., Jackson, J.M., Mauersberger. R. 1991, Astr. Ap. 241, L33

Nguyen-Q-Rieu, Nakai, N., Jackson, J.M. 1989, Astr. Ap. 220, 57

Lester, D.F., Carr, J.S., Joy, M., Gaffney, N. 1990, Ap. J. 352, 544

Seaquist, E.R., Bell, M.B. 1990, Ap.J. 364, 94

\section{QUESTIONS AND ANSWERS}

M.Guelin: In view of the small number of galaxies observed (and the way they were selected e.g. NGC 253 and M 82), I think it is preliminary to speak of "underabundances" of species such as $\mathrm{SiO}$ and HNCO. A pair of excitation problems, these species are detected only in few clouds in our own Galaxy; their non-detection in an external galaxy seems thus "normal".

C.Henkel: Since the extragalactic $H_{2}$ column densities may be uncertain and since it is difficult to detect some of the 'rare' species in galactic sources, it would indeed be preliminary to speak of 'underabundances' relative to galactic clouds. The term was used here exclusively in the context of comparing abundances between NGC 253 and M 82 .

R.Opher: If we would make the exact same analysis that you made for the 'other' molecules, to $\mathrm{CO}$ abundances, wouldn't we obtain an error in the Hydrogen abundance of more than $30 \%$ ?

C.Henkel: The $\mathrm{H}_{2}$ column densities are based on the integrated intensity of the CO $J=1-0$ line, using a standard conversion ratio derived from the galactic disk. This analysis differs from that used for the 'other molecules'. However, even if the applied conversion ratio is correct and does not vary, say with galactic radius, uncertainties may be $>30 \%$. This is perhaps most obvious in the case of Arp 220, where the CO based $\mathrm{H}_{2}$ column density refers to a region $300 \mathrm{pc}$ in diameter, while the $\mathrm{OH}$ maser arise from $\sim 1$ pc sized hotspots. 